differentiate into Th2 effectors. In contrast, naive $\mathrm{T}$ cells are capable of differentiating into Th2 cells with appropriate stimulation suggesting that the bias in Th1 differentiation of most RA patients is acquired during $\mathrm{T}$ cell maturation presumably in response to antigenic stimulation.

\section{THU0118 SEQUENTIAL EP1 AND EP4 REGULATION IN A RABBIT MODEL OF ANTIGEN-INDUCED ARTHRITIS}

I Diez-Ortego, R Largo, 0 Sanchez-Pernaute, R Guerrero, MJ Lopez-Armada, I Palacios, J Egido, G Herrero-Beaumont. Inflammation Research Unit, Fundacion Jimenez Diaz, Madrid, Spain

\subsection{6/annrheumdis-2001.995}

Background Prostaglandin E2 (PGE2) exerts its actions through the binding of the high affinity receptors EP. There are 4 isoforms of EP receptors with extracellular domains highly conserved, each coupling different intracellular transduction systems. Distribution of EP subtypes in the target tissues is therefore central to the pathways elicited by PGE2.

Objectives The aim of this work was to study the regulation of EP1 and EP4 receptors in the inflamed synovial tissue.

Methods We have studied different time periods in a rabbit model of antigen-induced arthritis: 24,48 and $72 \mathrm{~h}$ (acute phase) and 1, 2 and 3 weeks (chronic phase). Knee synovial fluid (SF) volume and white cell count were measured. Total RNA was extracted from the synovial membranes and expression of EP1 and EP4 receptors was studied by RT-PCR techniques. A fraction of synovial tissue was fixed and embedded in paraffin for morphological studies.

Results At the acute stages of the disease the animals depicted a synovial effusion that peaked at $48 \mathrm{~h}$. The highest white cell infiltration was found at $24 \mathrm{~h}$ and was dominated by neuthophils; at $72 \mathrm{~h}$ neutrophils and mononuclear cells showed similar concentrations in the SF. Al longer time-periods only half of the cases showed a synovial effusion, of smaller proportion. The acute histopathologic lesions consisted of leakage of plasma elements with prominent neutrophil invasion. At $72 \mathrm{~h}$ lining hyperplasia developed and there was a switch to mononuclear cell infiltration at the subintima. At further stages, fibrotic changes and increases vascularisation were also found. EP1 receptor expression was up-regulated at every point of study, when compared to controls. EP1 expression increased in a time dependent fashion, peaking at 2 weeks (14-fold vs. control). On the other hand, healthy controls expressed moderate EP4 levels, which were dramatically reduced at the acute stage of the disease $(90 \%$ reduction at $72 \mathrm{~h}$ ). At the latest periods studied, there was a normalisation and even slight up-regulation of EP4 expression.

Conclusion Our data may suggest that EP1 receptor mediates some of the PGE2 actions in experimental arthritis. This actions do not only include plasma leakage and activation of early inflammatory mediators, as EP1 up-regulation continues to increse when these typical features of acute arthritis are no longer evident. EP4 down-regulation at the early stages of the disease may suggest that this molecule do not participate in oedema and neutrophil activation induced by PGE2 in this experimental model. Restoring of the balance between PGE2 receptors in arthritis could be of help for the control of the inflammatory reaction.
THU0119 RHEUMATOID ARTHRITIS MYELOID CELLS EXPRESS AN INTRINSIC ABNORMAL CAPACITY FOR ACTIVATION

R Müller, A Skapenko, J Wendler, M Grünke, JR Kalden, H Schulze-Koops. Department of Internal Medicine III, University of Erlangen-Nuremberg, Erlangen, Germany

10.1136/annrheumdis-2001.996

Background Evidence suggests that monocyte precursor cells in rheumatoid arthritis (RA) might become partially activated in the bone marrow or the peripheral blood before they enter the rheumatoid synovium. However, whether this is the result of proinflammatory cytokines leaking from the inflamed synovium into the circulation or rather a functional abnormality of myeloid cells has not yet been conclusively delineated.

Objectives To test the hypothesis that monocytes in RA might express an intrinsic abnormality for activation, we investigated the function and the phenotype of peripheral blood monocytes from patients with RA and healthy controls in vitro.

Methods Monocytes were isolated by negative selection from patients with active, early RA and from patients with long standing RA under effective anti-inflammatory therapy with disease modifying antirheumatic drugs (DMARDs). A cell culture system was employed that permitted the differentiation of monocytes to macrophages. Expression of surface molecules indicative of activation and/or differentiation was assessed by flow cytometry.

Results No differences were detected for the expression of HLADR, CD14, CD40, CD68, CD80 and CD86 on freshly isolated monocytes between patients and controls. During differentiation to macrophages, the expression of HLA-DR, CD14 and CD80 was increased in RA monocytes. Interestingly, patients with untreated, active early disease manifested an even higher propensity to upregulate HLA-DR during differentiation compared to patients with long standing disease under effective anti-inflammatory therapy. In contrast, the expression of CD40, CD68 and CD86 was reduced in myeloid cells from RA patients during differentiation. This feature was more pronounced in patients with long standing disease, but was not correlated with clinical activity.

Conclusion The data indicate that monocytes from RA patients might have an intrinsically increased ability for activation during differentiation. This ability might contribute to clinical disease activity and emphasises the systemic nature of the disease. The data further suggest that long standing disease might alter the functional phenotype of circulating monocytes. However, the contribution of continuous treatment with DMARDs to this phenomenon remains to be elucidated.

\section{THU0120 POLYMORPHONUCLEAR NEUTROPHIL NADPH OXIDASE PRIMING AND ACTIVATION IN RHEUMATOID JOINTS: CORRELATION WITH DISEASE ACTIVITY AND SYNOVIAL CONCENTRATIONS OF TNF, IL-8 AND GM-CSF}

${ }^{1} \mathrm{G}$ Hayem, ${ }^{2} \mathrm{~J}$ El Benna, ${ }^{2} \mathrm{M}$ Fay, ${ }^{2} \mathrm{~S}$ Chollet-Martin, ${ }^{1} \mathrm{O}$ Meyer, ${ }^{2} \mathrm{MA}$ Gougerot-Pocidalo. ${ }^{1}$ Rheumatology Department; ${ }^{2}$ INSERM U479, Bichat Hospital, Paris, France

10.1136/annrheumdis-2001.997

Background Superoxide production by polymorphonuclear neutrophil (PMN) participates in the rheumatoid joint lesions and mainly depends on NADPH oxidase activation. Tumour necrosis factor (TNF), interleukin-8 (IL-8) and granulocyte/macrophage colony stimulating factor (GM-CSF) have a priming effect on NADPH oxidase, depending on the phosphorylation of p47phox, a major cytosolic component of NADPH oxidase. 